\title{
INFARTO AGUDO DO CEREBELO SIMULANDO TUMOR DA FOSSA POSTERIOR
}

\author{
JOSE ALBERTO GONCALVES DA SILVA *- ERASMO BARROS DA SILVA** \\ CARLOS AGRIPINO BRANCO***
}

\begin{abstract}
RESUMO - Um homem de 58 anos foi acometido subitamente de cefaléia, vômitos, vertigens e incoordenação dias extremidades esquerdas. A tomografia computadorizada do crânio evidenciou área hipodensa no hemisfério cerebelar esquerdo, cujo exame anátomo-patológico mostrou tratar-se de infarto cerebelar.
\end{abstract}

PALAVRAS CHAVE: cerehelo, infarto agudo, tratamento cirúrgico.

Acute cerebellar infarction presenting as a posterior fossa tumor.

SUMMARY - We report a case of space-occupying infarction of the left cerebellar hemisphere with occlusive hydrocephalus and left to right shift of the fourth ventricle. The patient, a 58 years old man, underwent shunting and decompressive craniectony of the posterior fossa and survived without neurological deficits.

KEI WORDS: cerebellum, acute infarction, surgical treatment.

Processos expansivos agudos da fossa posterior, especialmente hematomas intracerebelares $\mathrm{e}$ infartos isquêmicos do cerebelo, apresentam controvérsias quanto ao emprego do tratamento. Inicialmente, o diagnóstico definitivo de hemorragia ou infarto do cerebelo era, na maior parte dos casos, estabelecido em estudos necroscópicos ou através de craniectomia exploradora da fossa posterior. Somente após a introdução da tomografia computadorizada do crânio (TC) tornou-se possivel, na quase totalidade dos casos, o diagnóstico diferencial, de modo seguro e rápido, entre hemorragia e infarto cerebelares bem como entre outras patologias da fossa posterior. Em 1956, Fairburn e Oliver, segundo Auer e col.1, e Lindgren 9 publicaram primordialmente a descompressão da fossa posterior como tratamento de escolha nos infartos do cerebelo. Desde então passou a haver divergências entre o emprego do tratamento clínico e do cirúrgico, variando a técnica utilizada de drenagem ventricular aberta, ventriculoperitoniostomia, descompressão da fossa posterior ou a combinação de técnicas. A partir das publicações de Fairburn e Oliver e de Lindgren, a literatura passou a se enriquecer $2,4-7,11,12,14,15$.

A publicação do presente caso se baseia na raridade da patologia e no fato de não termos encontrado registro semelhante na literatura brasileira.

\section{OBSERVACXO}

RAN, paciente com 58 anos de idade, do sexo masculino, branco, paraibano, internado em 23-09-90 no Hospital São Vicente de Paulo, registro 6217. História de instalação aguda, iniciada há 5 dias com cefaléia intensa, vômitos sucessivos, vertigens e incoordenação dos

* Chefe do Serviço de Neurologia e Neurocirurgia do Hospital Santa Isabel; ** Professor Adjunto de Neurologia da Universidade Estadual da Paraíba (UFPB); *** Médico Ortalmologista, Chefe da Clínica São Camilo.

Dr. J. A. Gonqalves da Silva - Av. Minas Gerais 1150 - 58030 - João Pessoa PB - Brasil. 
membros esquerdos. O exame clínico-neurológico mostrou: pressão arterial 180x130mmHg; sonolência acentuada; marcha atáxica, com duplo apoio; paralisia do nervo abducente esquerdo (E); nistagmo horizontal e vertical para cima e para baixo; sinal de Babinski bilateral; ataxia vermiana e do hemisfério cerebelar $E$, observando-se, na prova indice-nariz, dismetria, tremor intencional e decomposição dos movimentos. A TC evidenciou lesão hipodensa com diâmetro de $6 \times 5 \mathrm{~cm}$ no hemisfério cerebelar $\mathrm{E}$, compressão e deformidade do quarto ventrículo e hidrocéfalo. Foi sugerida a possibilidade de astrocitoma cerebelar. Em 23-08-90, o paciente foi submetido a ventriculoperitoniostomia com melhora imediata da ataxia, mas persistindo sonolência acentuada. Em 25-09-90, o paciente foi submetido a craniectomia da fossa posterior à $\mathbf{E}$ com aspiração do tecido cerebelar necrosado. $O$ exame anátomo-patológico evidenciou infarto isquêmico com edema intersticial. Em 28-09-90, teve alta com melhora acentuada da ataxia cerebelar e com regressão do estado de sonolência. Em 08-04-91, o seguimento revelou regressão total de todos os sintomas e sinais neurológicos, readquirindo o paciente suas funções laborativas de modo integral.

\section{COMENTARIOS}

O termo infarto cerebelar se refere a lesão isquêmica do cerebelo sem envolvimento primário do cérebro ou tronco cerebral 2,14. Goodhart e Davison, segundo Cioffi e col.2, admitiram em 1936 que o infarte do cerebelo pode ser fatal na fase aguda, quando houver compressão concomitante do tronco cerebral. Esta condição clínica foi raramente diagnosticada antes da introdução da $T C$, que proporciona não só o diagnóstico diferencial entre hematoma intracerebelar e infarto do cerebelo mas, também, permite observar a evolução do quadro clínico por tomografias sequenciais. Entre os tatores etiológicos sobressai a aterosclerose com suas múltiplas consequências. Segundo Heros $6,50 \%$ dos casos de infarto cerebelar provêm de êmbolos cardíacos. Hipertensão arterial, infarto do miocárdio, insuficiência cardíaca e diabete são outros fatores de risco observados nos pacientes 1,6,8,10-12. Nosso paciente, por ocasião da admissão, evidenciava pressão arterial de $180 \times 130$.

Em relação à patologia, cerca de $85 \%$ dos infartos cerebelares ocorrem no território da artéria cerebelar posterior e inferior, o que corresponde à porção medial e inferior de um hemisfério cerebelar 6 . Oclusão uni ou bilateral das artérias vertebrais ocorre em $50 \%$ dos casos 6 . Sypert e Alvord 14 observaram, em $65 \%$, oclusão recente de uma artéria vertebral como causa de infarte extenso do cerebelo. Do mesmo modo, os infartos cerebelares assintomáticos se acham frequentemente associados a oclusão da artéria vertebral ipsilateral 14. Sypert e Alvord 14 publicaram estudo clínico-patológico minucioso sobre o infarto do cerebelo, baseados em 28 casos não complicados. Esses autores dividem os infartos em complicados e não complicados; o infarto cerebelar complicado é caracterizado pelo comprometimento isquêmico tanto do cerebelo quanto do tronco cerebral. Ebeling e col. 3 distinguem dois grupos de pacientes, de acordo com a anamnese, nos processos agudos da fossa posterior: o grupo 1 engloba os pacientes com evolução lenta e progressiva e que desenvolvem piora aguda; no grupo 2 se acham os pacientes que, após início agudo do quadro clínico, desenvolvem evolução fulminante. Os pacientes do grupo 1 apresentam sintomas e sinais iniciais e aqueles de alarme, enquanto os do grupo 2 exibem apenas sintomatologia de alarme. A sintomatologia inicial é caracterizada por náusea, vômitos, cefaléia, vertigens e ataxia, enquanto a sintomatologia de alarme, que evidencia a compressão do tronco cerebral, caracteriza-se por meningismo, distúrbios da consciência, lesão de nervos cranianos, lesão piramidal, respiração e circulação patológicas e decerebração. A sintomatologia de alarme inicia, portanto, o processo grave de descompensação cerebral.

No caso em estudo, o paciente apresentava sintomatologia inicial caracterizada por cefaléia, vômitos, vertigens e incoordenação cerebelar, além de sinais de compressão do tronco cerebral como paralisia do nervo abducente esquerdo, nistagmo, sinal de Babinski bilateral e estado de sonolência acentuada.

O aumento da massa cerebelar, para poder comprimir o tronco cerebral, é provocado por edema intra e extracelular acentuado, nas grandes áreas infartadas do cerebelo, de acordo com Sypert e Alvord 14. Estes autores admitem que a extensão do infarte é importante, pois edema intenso do cerebelo não foi observado em infartos que comprometeram menos do que um terço de um hemisfério cerebelar. No caso em estudo, o edema do hemisfério cerebelar esquerdo provocou sinais de compressão do tronco cerebral e desvio do quarto ventrículo com formação de hidrocéfalo. 
O prognóstico dos processos agudos da fossa posterior, especialmente dos hematomas e infartos cerebelares, depende de vários fatores. A extensão do hematoma cerebelar ou do infarto do cerebelo irá determinar o tipo de evolução do quadro clínico. Segundo Ebeling e col.3, os hematomas com diâmetro horizontal maior do que $3 \mathrm{~cm}$ (von Little, Lui e Salazar) e segundo Mitchell maior do que $5 \mathrm{~cm}$, apresentam prognóstico pior com mortalidade e morbidade mais elevadas quando se emprega o tratamento clínico isoladamente. O nível de consciência tem importância fundamental quanto ao prognóstico. Quanto mais grave for o distúrbio da consciência, tanto pior será a evolução do paciente 1,3,6. Por outro lado, o tempo de compressão do tronco cerebral, até ser realizada a descompressão da fossa posterior, terá influência decisiva na recuperação ou não do paciente 3 .

O emprego do tratamento clínico deve ser indicado em casos de infartos que comprometam menos que um terço de um hemisfério cerebelar e nos quais os pacientes se achem sem distúrbios da consciência, com quadro clínico estável 1,3,6-8. Greenberg e col.4 contra-indicam o tratamento clínico nos casos de infartos extensos. Nestes casos verificaram mortalidade de $55 \%$ e, nos pacientes em coma, mortalidade de $80 \%$. Momose e Lehrich 11 trataram 13 pacientes com infarto cerebelar: faleceram 4 pacientes tratados clinicamente e 9 foram submetidos a craniectomia da fossa posterior, dos quais um faleceu.

O tratamento cirúrgico do infarto cerebelar compreende a drenagem ventricular externa, a derivação ventrículo-peritoneal ou a craniectomia da fossa posterior. O método de drenagem do líquido cefalorraqueano (LCR) - drenagem ventricular externa ou «shunt» - quando usado isoladamente, piorou o prognóstico de uma parte dos pacientes em várias casuísticas 1-3. A piora, na evolução destes pacientes, foi imputada à instalação de hérnia transtentorial ascendente, entre outros por Epstein $e$ Murali, Horwitz, Raimandi e Tomita (citados por Ebeling e col.3). A derivação do LCR isoladamente estaria indicada nos casos com hidrocéfalo e na ausência de sinais de compressão do tronco cerebral 3.

A craniectomia da fossa posterior, método introduzido por Lindgren 9 em 1956 , é o método utilizado pela maioria dos autores 1-3,6,9-11,15 nos casos de hematomas intracerebelares com diâmetro transverso maior do que $3 \mathrm{~cm}$ e nos infartos que comprometem mais que um terço de um hemisfério cerebelar. $O$ dado clínico mais importante para a indicação cirúrgica é o comprometimento progressivo da consciência, que traduz compressão do tronco cerebelar.

No presente estudo, a indicação da derivação do LCR fundamentou-se na presença de hidrocéfalo. Como no pós-operatório, apesar da melhora da sintomatologia observada, persistiu o estado de sonolência, optamos então pela descompressão da fossa posterior. Através desta operação, foi evitada a possivel instalação de hérnia transtentória ascendente bem como encurtamos o tempo da compressão cerebelar sobre o tronco cerebral, o que comumente agrava o prognóstico. grafia.

Agradecimento - Agradecemos ao Dr. Clemente Augusto Pereira a aquisição da biblio-

\section{REFERENCIAS}

1. Auer LM, Auer T, Sayama I. Indications for surgical treatment of cerebellar haemorrhage and infarction. Acta Neurochir 1986, 79:74-79.

2. Cioffi FA, Bernini FP, Punzo A, D'Avanzo R. Surgical management of acute cerebellar infarction. Acta Neurochir 1985, 74:105-112.

3. Eoeling $U$, stricker $K$, Reulen $H J$, Hase $U$. Akute Raumforderungen der hinteren Schaedelgrube: Klinik, Therapie und Ergebnisse. In Hase U, Reulen HJ (eds): Die akute Raumforderung in der hinteren Schaedelgmube. Wien: Ueberreuter, 1988, p $137-152$.

4. Greenberg J, Skubick D, Shenkin H. Acute hydrocephalus in cerebellar infart and hemorrhage. Neurology 1979, 29:409-413.

5. Hase U, Reulen HJ. Die akute Raumforderung in der hinteren Schaedelgrube. Wien: Ueberreuter Wissenschaft, 1988.

6. Heros RC.' Cerebellar hemorrhage and infarction. Stroke 1982, 13:106-109.

7. Hinshaw DB Jr, Thompson JR, Hasso AN, Casselman ES. Infarctions of the brainstem and cerebellum: a correlation of computed tomography and angiography. Radiology 1980. $187: 105-112$. 
8. Kazner E, Sprung C, Kutschenreuther R, Grumme T, Lanksch W. Differenzierte Behandlung des Kleinhirninfarktes. In Hase $U$, Reulen HJ (eds): Die akute Raumforderung in der hinteren Schaedelgrube. Wien: Ueberreuter 1988, p 105-113.

9. Lindgren SO. Infarctions simulating brain tumours in the posterior fossa. $J$ Neurosurg 1956, $13: 575-581$.

10. Mokry M, Auer LM. Blutungen und Infarkte des Klainhirns: konservative oder operative Behandlung?' In Hase U, Reulen HJ (eds): Die akute Raumforderung in der hinteren Schaedelgrube. Wien: Ueberreuter, 1988, p 125-131.

11. Momose KJ, Lehrich JR. Acute cerebellar infarction presenting as a posterior fossa mass. Radiology 1973, 109:343-352.

12. Norris JW, Eisen AA, Branch CL. Problems in cerebellar hemorrhage and infarction. Neurology 1969, 19:1043-1050.

13. Perneczky A, Ungersboeck $K$. Blutungen und Malazien in der hinteren Schaedelgrube: chirurgische oder konservative Therapie? In Hase $U$, Reulen $H J$ (eds): Die akute Raumforderung in der hinteren Schaedelgrube. Wien: Ueberreuter, 1988, p 114-124.

14. Sypert GW, Alvord EC Jr. Cerebellar infarction: a clinico-pathological study. Arch Neurol 1975, 32:357-363.

5. Wood MW, Murphey F. Obstructive hydrocephalus due to infarction of a cerebellar hemisphere. J Neurosurg 1969, 30:260-263. 